\title{
Equilibrium and Renegotiation in Delegation Games
}

\author{
Michele Polo \\ IGIER and Bocconi University \\ Milan
}

\author{
Piero Tedeschi \\ Department of Economics \\ University of Padua *
}

\begin{abstract}
We propose a general formal structure for symmetric information delegation games that encompasses many existing economic applications in the fields of oligopoly theory, the theory of the firm, strategic trade policy and international political economy. We prove that all individually rational allocations are implementable in delegation games with non separable utility. Secondly, we show that contract renegotiation and non observable contracts have similar effects only in particular cases. We prove that all the equilibria obtained when renegotiation is excluded are implementable as renegotiation proof equilibria, provided that the side transfer technology implies a dead-weight loss increasing in the size of the transfer.
\end{abstract}

JEL: C72, L22

Keywords: Delegation, Renegotiation

\section{Introduction}

Delegation games represent a strategic situation in which many agencies interact non cooperatively. In this paper we consider two stage delegation games in which the principals choose a compensation scheme for their agents, while these latter play a game on behalf of the principals. The payoffs of all players are determined by the actions chosen by the agents. The principals can influence the outcome of the game indirectly, by shaping their own agent's reaction function through the design of an incentive scheme, which becomes public information once chosen ${ }^{1}$.

*Mailing address: Michele Polo, Dept. Economics, Bocconi University, Via Sarfatti 25, 20123 Milan, Italy, e-mail: michele.polo@uni-bocconi.it. Financial support is acknoledged from MURST (Italian University Department) "Rapporti di agenzia in organizzazioni pubbliche e private" (Michele Polo), from MURST "Teoria dei giochi e applicazioni" (Piero Tedeschi) and from Universita' Bocconi "Fondo per il sostegno della ricerca di base" (both authors). We wish to thank Pier Paolo Battigalli, Jacques Cremer, Pier Angelo Mori and Guido Tabellini. Usual disclaimers apply.

${ }^{1}$ Alternatively, one could think of the principals as choosing the type of their own agent. 
This framework has been applied to a variety of economic problems, a (probably incomplete) list of which includes managerial contracts and vertical relations in oligopoly, strategic trade policy and international political economy ${ }^{2}$. The first topic was first explored, largely at a qualitative level, by Vickers' (1985) seminal paper, and further developed by Fershtman and Judd (1987), Sklivas (1987), Polo and Tedeschi (1992) and Katz (1991). The effects of strategic delegation on the internal architecture of firms is analized in Koray and Sertel (1989) for what concerns hierarchical relations and in Polo and Tedeschi (1990) with reference to the firm divisional structure. Vertical contractual relations are studied in Kühn (1990). Delegation games proved to be very useful for the analysis of policy design issues. For instance, the theory of strategic trade policy was originated by two seminal contributions by Brander and Spencer (1983), (1985) and developed by a series of paper, examples of which are Eaton and Grossman (1986), Laussel (1992) and Maggi (1991). Finally, an example of international political economy models is Persson and Tabellini (1992). It is worth noting that this literature has been developed almost completely through examples, with very few general results. Exceptions are Fershtman, Judd and Kalai (1991), Katz (1991) and Caillaud et al. (1993). The first proves a Folk Theorem in delegation games with separable utility. Katz shows that most of the strategic effect of delegation is lost if contracts are unobservable. Caillaud et al. prove that with asymmetric information delegation has strategic effects, even if secret renegotiation is allowed. However, to obtain this result, it is necessary to make specific assumptions about strategic complementarity and on the direct effect of the opponents' action on the principals' welfare. The results in Caillaud et al. (1993) were anticipated by examples examined in Katz (1991) and in Fershtman and Judd (1986).

Our first aim is to prove the existence and to characterize the set of equilibria in a unified formal structure, which can encompass most of the quoted 2-agency models with symmetric information ${ }^{3}$. In this framework we prove a Folk Theorem for delegation games. The result is more general than the one obtained in Fershtman, Judd and Kalai (1991), since we allow for non separable utilities. Moreover, the set of equilibrium allocations includes all the individually rational allocations and not only the Pareto efficient ones, as in that paper. The proof of the Folk Theorem uses a preliminary result, that we define Stackelberg Property, which shows that the incentive compatibility constraint of the agent is not binding for his own principal ${ }^{4}$. Hence, in a subgame perfect equilibrium, each principal chooses a contract which induces the most preferred allocation on the reaction function of the rival agent, once taken into account the participation contraint of her own agent, i.e. each principal acts as a Stackelberg leader with respect to all agents.

The Stackelberg Property allows us to show why the high multiplicity of equilibria implied by the Folk Theorem is endemic in these models. The explanation is intuitively as follows. Suppose that the equilibrium is an internal maximum for both agencies and that

\footnotetext{
${ }^{2}$ Moreover, delegation games have close relations with supply function equilibria - Klemperer and Meyer (1989)

${ }^{3}$ Almost all the applications, as well as the theoretical papers by Fershtman Judd and Kalai (1991), Katz (1991) abd Caillaud et al. (1993), deal with 2-agency delegation games.

${ }^{4}$ In what follows we shall conventionally use the female for the principal and the male for the agent.
} 
the maximizing action of both agents is unique in the neighborhood of the equilibrium. The equilibrium imposes only local restrictions on the compensation schemes, i.e. the indifference curve of each principal must be tangent to the best reply function of the other agent ${ }^{5}$. If the principals have enough degrees of freedom in shaping the reaction function of their own agent, almost any allocation can be sustained as an equilibrium of the delegation game. While in some applications there exists a unique equilibrium, e.g. Fershtman and judd (1987) and Sklivas (1987), this result is obtained by imposing strong restrictions on the set of feasible contracts. Polo and Tedeschi (1991) prove in the same setting the existence of a high multiplicity of equilibria if the set of feasible contracts is sufficiently enlarged.

The second aim of this paper relates to renegotiation and observability of contracts. It is often argued that the relevance of delegation games is questionable, since their main results hold only under very restrictive assumptions, i.e. that agents' contracts are public information and not renegotiable. When one of these assumptions is removed, it can be proved that the wide multiplicity of equilibria disappears, often remaining with the set of equilibria of the game without delegation. To illustrate this point, suppose that the payoffs of the agents depend only upon their own compensation scheme, which is public information. In that case each player can compute the best reply functions and the Nash equilibrium of the agents' game. Suppose now that principal $i$ can secretly renegotiate the compensation to agent $i$. Since the other agent cannot observe principal $i$ s deviation, he cannot react. This implies that principal $i$ will compute the payoff of her deviation from the proposed strategy profile holding the other agent's action, and not his best reply function, constant. Hence, when designing the optimal (secret) side contract each principal solves a problem that is very similar to the one in which the contract is private information. Katz (1991) argues that the non-observability of contracts and the possibility of secret renegotiation produce the same effects in delegation games, since in both cases the strategic dimension of delegation is almost completely lost, at least under symmetric information. With asymmetric information the same work by Katz (1991) and especially that of Caillaud et al. (1993) prove that delegation preserves a strategic effect under specific assumption, but a Folk Theorem cannot be proved.

Contrary to Katz's (1991) argument, and maintaining the assumption of symmetric information, we prove that renegotiation proof equilibria can induce a set of outcomes identical to that of the games where renegotiation is excluded. The crucial condition is that the side transfer technology implies a dead-weight loss increasing in the size of the side compensation. This assumption has been used with different motivations both in the theory of interest groups - Stigler (1971) - and in that of collusion in organizations - Tirole (1992), p. 152. In our case, by designing the main contract with a kink at the equilibrium allocation, the principals are able to make ex post renegotiation too costly

\footnotetext{
${ }^{5}$ Of course, also the second order conditions must be satisfied, which anyhow translate into weak restrictions on the compensation schemes. Moreover, the notion of indifference curves must be qualified, since in constructing them we need to take care of the participation constraint of the agent. Hence, the indifference curves imply the same payoff for the principal while maintaining the agent at the reservation utility.
} 
for themselves. This result holds for increasing, but arbitrarily small, extra-costs of the side contract.

The paper is organized as follows. In the next section we set up the model. In the third section we prove a Folk Theorem for delegation games. Section 4 modifies the model in order to take into account the issue of renegotiation proofness. In section 5 we derive the main propositions regarding renegotiation proof equilibria. Concluding remarks follow.

\section{2-Agencies Delegation Games}

In this section we set up the main features of a 2-agency delegation game, stating and discussing the relevant assumptions. Informally, this class of games describes the strategic interaction between two agencies, each composed by one principal and one agent. The principals do not participate directly in the game, which is played on their behalf by the agents. However, the former can influence the outcome of the game by shaping the payoff of the latter. We first introduce most of the assumptions and then discuss them briefly.

Assumption 1 (Players). The set of players is $I=\left\{p^{1}, p^{2}, a^{1}, a^{2}\right\}$ and can be partitioned in two subsets: $P=\left\{p^{1}, p^{2}\right\}$, the set of principals, and $A=\left\{a^{1}, a^{2}\right\}$, the set of agents. Players $p^{i}$ and $a^{i}$ constitute agency $i$.

Assumption 2 (Agents' actions). $S^{i} \subseteq \Re$ is the compact action set of agent $a^{i} \in A$ and $s^{i}, s^{i} \in S^{i}$, is its generic element. We adopt the usual notation, $S=S^{1} \times S^{2}$, with generic element $s$.

Assumption 3 (Principals' utility). $u_{p}^{i}: S \times \Re \rightarrow \Re^{+}$is the utility function of $p^{i}$ with the following properties: $u_{p}^{i} \in C^{2}$, it is strictly quasi-concave in $\left\{s^{i}, m^{i}\right\}$ and strictly decreasing in $m^{i}$, which is agent $i$ 's compensation.

Assumption 4 (Agents' utility). $u_{a}^{i}: S \times \Re \rightarrow \Re^{+}$is the utility function of $a^{i}$ with the following properties: $u_{p}^{i} \in C^{3}$, it is strictly quasi-concave in $\left\{s^{i}, m^{i}\right\}$ and strictly increasing in $m^{i}$, for any $s \in S$.

Assumption 5 (Individual rationality constraint). The outside options for the principals and the agents are respectively: $\bar{u}_{p}$ for all $p^{i}$ and $\bar{u}_{a}$ for all $a^{i}$. Moreover define the principal's min-max utility $u_{m M}^{i}$ as $u_{m M}^{i}=\min _{s^{j}} \max _{s^{i}, m^{i}} u_{p}^{i}\left(s, m^{i}\right)$, subject to $u_{a}^{i}\left(s, m^{i}\right) \geq \bar{u}_{a}$. The set of individually rational allocations for agency $i$ is:

$$
S_{0}^{i}=\left\{s \in S \mid \exists m^{i}: u_{a}^{i}\left(s, m^{i}\right) \geq \bar{u}_{a}, u_{p}^{i}\left(s, m^{i}\right) \geq \max \left(\bar{u}_{p}, u_{m M}^{i}\right)\right\}
$$

for $i=1,2$, while the set of individually rational allocations is $S_{0}=S_{0}^{1} \cap S_{0}^{2}$. We assume that $S_{0}$ is non empty. 
In Assumption 1 we consider simple agencies with only one principal and one agent. Assumption 2 implies that each agent's action space is an interval on the real line. The utility of each principal and agent (Assumptions 3 and 4) depends upon the actions taken by the agents; $m^{i}$ is a transfer payment from $p^{i}$ to $a^{i}$. Note that we do not assume separable utility in income and actions, neither in the principals', not in the agents' utility function as it is usually done in this literature. Moreover, to obtain a non trivial solution of the game, we impose further restrictions on the utility functions, concerning the individual rationality constraints for principals and agents (Assumption 5). The individual rationality constraint for the principal implies that the utility must be at least equal to the largest value between the outside option and the min-max. In fact, the principal can never be forced to go below these two levels of utility. Finally, we consider a two stage game, characterized by the following timing.

Assumption 6 (Timing of the game). The utility functions of all principals and all agents are common knowledge from the beginning.

$t_{1}:$ Each principal proposes a contract $\mu^{i}, \mu^{i}: S \rightarrow \Re$, to the agent, who can accept it or not. If $a^{i}$ refuses it, the game is over, otherwise if continues;

$t_{1}^{\prime}:$ All contracts become public information;

$t_{2}:$ Each $a^{i}$ chooses an action $s^{i} \in S^{i}$;

$t_{3}: s$ is observed (and verifiable) and all contracts are implemented.

Thus, the principals have all the bargaining power with respect to their own agents. Moreover, Assumption 6 above implies a symmetric information game, contrary to the usual agency problem. This is a common assumption in the literature on delegation games, with few exceptions, notable examples of which are Caillaud et al. (1993), Fershtman and Judd (1986) and Katz (1991). The following two assumptions complete the description of the game.

Assumption 7 (Principals' strategy). The strategy set of principal $p^{i}$ is the set $M^{i}$ of all functions $\mu^{i}: S \rightarrow \Re$. The set of principals' strategy profiles is $M=M^{1} \times M^{2}$, with generic element $\mu$.

The assumption about the contract form may seem rather unrealistic. In fact, we rarely observe contracts conditioned on the actions of both agents. However, the assumed contract form does not seem very restrictive, if we interpret it as a recuced form of more realistic ones ${ }^{6}$.

\footnotetext{
${ }^{6}$ In the managerial contracts literature, for instance, managers' compensations are conditioned to some linear combination of revenues, costs and rival firms' profits. This is equivalent to conditioning the compensation to the managers' actions. In asymmetric information delegation games this assumption would obviously be much less innocuous.
} 
Assumption 8 (Agents' strategy). A strategy for agent $a^{i}$ is the choice of a function $\sigma^{i}: M \rightarrow S^{i} . \Sigma^{i}$ is the strategy set of agent $a^{i}$ and the set of all agents' strategy profiles is $\Sigma=\Sigma^{1} \times \Sigma^{2}$, with generic element $\sigma$.

Given Assumptions from 1 to 8, a two stage delegation game is

$$
{ }^{d}=\left\{\{P, A\},\{M, \Sigma\},\left\{u_{p}^{i}, u_{a}^{i}\right\}_{i=1,2},\left\{\bar{u}_{p}, \bar{u}_{a}\right\}\right\}
$$

In the next section we analyse the equilibria in delegation games.

\section{A Folk Theorem for Delegation Games}

In the previous section we have defined a two-stage game of symmetric information, whose appropriate equilibrium concept is subgame perfection. Consequently, the equilibrium analysis requires to work backward, beginning from the last stage game played by the agents.

$\tilde{\sigma}^{i}\left(s^{j} ; \mu^{i}\right)$ is an implementable reply function for $a^{i}$ if there exists a $\mu^{i}$ such that

$$
\tilde{\sigma}^{i}\left(s^{j} ; \mu^{i}\right) \in \arg \max _{s^{i}} u_{a}^{i}\left(s^{i}, s^{j} ; \mu^{i}\left(s^{i}, s^{j}\right)\right)
$$

$\tilde{\sigma}^{i}(\cdot)$ identifies a function that principal $p^{i}$ can implement as $a^{i}$ s best reply. The Nash equilibrium in the last stage game among agents can now be defined in the traditional way. A Nash equilibrium in the 2-agent game at $t_{2}$ is a set of functions

$$
\hat{\sigma}(\mu)=\left\{\hat{\sigma}^{i}(\mu), \hat{\sigma}^{j}(\mu)\right\}
$$

such that

$$
\hat{\sigma}^{i}(\mu)=\tilde{\sigma}^{i}\left(\hat{s}^{j}, \mu^{i}\right) \quad \text { and } \quad u_{a}^{i}(\hat{s} ; \mu(\hat{s})) \geq \bar{u}_{a}
$$

for $i=1,2$, where $\hat{s}^{i}=\hat{\sigma}^{i}(\mu)$ and $\hat{s}=\hat{\sigma}(\mu)$.

A subgame perfect equilibrium in , ${ }^{d}$, (SPE), is given by two sets of functions $\hat{\mu}$ and $\hat{\sigma}$ such that

$$
u_{p}^{i}\left(\hat{\sigma}(\hat{\mu}), \hat{\mu}^{i}(\hat{\sigma}(\hat{\mu}))\right) \geq u_{p}^{i}\left(\hat{\sigma}\left(\mu^{i}, \hat{\mu}^{j}\right), \mu^{i}\left(\hat{\sigma}\left(\mu^{i}, \hat{\mu}^{j}\right)\right)\right)
$$

for any $\mu^{i} \in M^{i}, i, j=1,2, i \neq j$ and where $\hat{\sigma}$ is a Nash equilibrium in the agents' game.

The following theorem establishes a preliminary and useful result.

Theorem 1 (Stackelberg Property): Necessary and sufficient condition for two set of functions $\hat{\mu}$ and $\hat{\sigma}$ to constitute a SPE is that, setting $\hat{s}=\hat{\sigma}(\hat{\mu})$ and $\hat{m}=\hat{\mu}(\hat{s}), \hat{s}$ and $\hat{m}$ are the solution of the following problem (S) for all $i$ : 


$$
\begin{array}{ccl}
\max _{s^{i}, m^{i}} & u_{p}^{i}\left(s, m^{i}\right) \\
\text { (S) } \quad \text { s.t. } & s \in S_{0}^{i} \\
& s^{j}=\tilde{\sigma}^{j}\left(s^{i} ; \mu^{j}\right)
\end{array}
$$

Proof: The proof is by contradiction. Notice that $\hat{s} \in S_{0}$, because the participation constraint must hold for all $i$. Moreover, since we maximize principals' utility, the individual rationality constraint of both agents will bind. Furthermore, in $S_{0}$ principals' individual rationality constraint is certainly satisfied. Suppose that $\hat{s}$ and $\hat{m}$ are the solution of problem (S) for all $i$, but $\hat{\mu}$ and $\hat{\sigma}$ are not a SPE. Since (S) must hold for all $i$, it is easy to check that $\hat{\sigma}$ is a Nash equilibrium in the agents' game. Therefore, if $\hat{\mu}$ and $\hat{\sigma}$ are not a SPE there must exist for at least one principal a profitable deviation, i.e. a contract $\overline{\bar{\mu}}^{i}$ such that the vector of contracts $\overline{\bar{\mu}}=\left\{\overline{\bar{\mu}}^{i}, \hat{\mu}^{j}\right\}$ induces a Nash equilibrium outcome for the last stage game $\overline{\bar{s}}=\left\{\overline{\bar{s}}^{i}, \overline{\bar{s}}^{j}\right\}=\left\{\tilde{\sigma}^{i}\left(\overline{\bar{s}}^{j}, \overline{\bar{\mu}}^{i}\right), \tilde{\sigma}^{j}\left(\overline{\bar{s}}^{i}, \hat{\mu}^{j}\right)\right\}$ that satisfies

$$
u_{p}^{i}\left(\overline{\bar{s}}, \overline{\bar{\mu}}^{i}(\overline{\bar{s}})\right)>u_{p}^{i}\left(\hat{s}, \hat{\mu}^{i}(\hat{s})\right)
$$

Hence $\hat{s}^{i}$ and $\hat{m}^{i}$ cannot be the solution of program (S). Now suppose that $\hat{\mu}$ and $\hat{\sigma}$ are a SPE but that $\hat{s}$ and $\hat{m}$ do not solve problem (S) for agency $i$. In this case there must exist a couple $\underline{\underline{s}}^{i}$ and $\underline{\underline{m}}^{i}$ such that

$$
\begin{array}{r}
u_{p}^{i}\left(\underline{\underline{s}}^{i}, \tilde{\sigma}^{j}\left(\underline{\underline{s}} i, \hat{\mu}^{j}\right), \underline{\underline{m}}^{i}\right)>u_{p}^{i}(\hat{s}, \hat{m}) \\
u_{a}^{i}\left(\underline{\underline{s}}, \hat{\sigma}^{j}\left(\underline{\underline{s}}, \hat{\mu}^{j}\right), \underline{\underline{m}}^{i}\right)=\bar{u}_{a}
\end{array}
$$

It is easy to prove that $u_{a}^{i}\left(\hat{s}, \hat{m}^{i}\right)=\bar{u}_{a}$. By continuity it is always possible to find an $\breve{m}^{i}>\underline{\underline{m}}^{i}$ such that

$$
\begin{array}{r}
u_{p}^{i}\left(\underline{\underline{s}}^{i}, \tilde{\sigma}^{j}\left(\underline{\underline{s}}^{i}, \hat{\mu}^{j}\right), \breve{m}^{i}\right)>u_{p}^{i}(\hat{s}, \hat{m}) \\
u_{a}^{i}\left(\underline{\underline{s}}^{i}, \tilde{\sigma}^{j}\left(\underline{\underline{s}}, \hat{\mu}^{j}\right), \breve{m}^{i}\right)>\bar{u}_{a}
\end{array}
$$

Therefore the principal can choose the following contract:

$$
\underline{\underline{\mu}}^{i}= \begin{cases}\breve{m}^{i} & \text { if } s^{i}=\underline{\underline{s}}^{i} \\ \hat{\mu}^{i}(s) & \text { otherwise }\end{cases}
$$

By construction this contract induces $\left(\underline{s}^{i}, \tilde{\sigma}^{j}\left(\underline{s}^{i}, \hat{\mu}^{j}\right)\right.$ as the equilibrium outcome of the agents game and therefore it is a profitable deviation for the principal. We conclude that $\hat{\mu}$ and $\hat{\sigma}$ cannot be a SPE.

Problem (S) is the usual Stackelberg problem in a sequential game. Theorem 1 shows that $a^{i}$ s incentive compatibility constraint is not binding for $p^{i}$. Hence, in a SPE, each 
principal acts as a Stackelberg leader with respect to the other agent, picking the most preferred allocation along the rival agent's best reply function, once taken into account the participation constraint of the agent.

This fact can be better appreciated if we use contracts in $C^{2}$. The first order conditions of problem $(S)$, being $\lambda^{i}$ the Lagrange multiplier of agent $i$ 's participation constraint, become

$$
\begin{aligned}
\frac{\partial u_{p}^{i}}{\partial s^{i}}+\frac{\partial u_{p}^{i}}{\partial s^{j}} \cdot \frac{\partial \tilde{\sigma}^{j}}{\partial s^{i}}+\lambda^{i}\left(\frac{\partial u_{a}^{i}}{\partial s^{i}}+\frac{\partial u_{a}^{i}}{\partial s^{j}} \cdot \frac{\partial \tilde{\sigma}^{j}}{\partial s^{i}}\right) & =0 \\
\frac{\partial u_{p}^{i}}{\partial m^{i}}+\lambda^{i} \frac{\partial u_{a}^{i}}{\partial m^{i}} & =0
\end{aligned}
$$

which jointly give:

$$
\frac{\partial \tilde{\sigma}^{j}}{\partial s^{i}}=-\frac{\frac{\partial u_{p}^{i} / \partial s^{i}}{\partial u_{p}^{2} / \partial m^{2}}-\frac{\partial u_{a}^{i} / \partial s^{i}}{\partial u_{a}^{i} / \partial m^{i}}}{\frac{\partial u_{p}^{i} / \partial s^{j}}{\partial u_{p}^{2} / \partial m^{2}}-\frac{\partial u_{a}^{i} / \partial s^{j}}{\partial u_{a}^{i} / \partial m^{2}}}
$$

Equation (3) has a nice geometric interpretation. Consider a contract $\bar{\mu}^{i}(s)$ which, in a neighborhood of the equilibrium, keeps $a^{i}$ s utility constraint at the reservation value, i.e. $u_{a}^{i}\left(\hat{s}, \bar{\mu}^{i}(s)\right)=\bar{u}_{a}$. The partial derivatives of the contract are

$$
\frac{\partial \bar{\mu}^{i}}{\partial s^{i}}=-\frac{\partial u_{a}^{i} / \partial s^{i}}{\partial u_{a}^{i} / \partial m^{i}} \quad \frac{\partial \bar{\mu}^{i}}{\partial s^{j}}=-\frac{\partial u_{a}^{i} / \partial s^{j}}{\partial u_{a}^{i} / \partial m^{i}}
$$

Principal $p^{i}$ s indifference curves, when agent $a^{i}$ is kept at the reservation utility, are therefore implicitly defined as $u_{p}^{i}\left(\hat{s}, \bar{\mu}^{i}(s)\right)=k$, with slope

$$
\left.\frac{\partial s^{j}}{\partial s^{i}}\right|_{u_{p}^{i}=k}=-\frac{\frac{\partial u_{p}^{i}}{\partial s^{i}}-\frac{\partial u_{p}^{i}}{\partial m^{2}} \cdot \frac{\partial u_{a}^{i} / \partial s^{i}}{\partial u_{a}^{i} / \partial m^{i}}}{\frac{\partial u_{p}^{i}}{\partial s^{j}}-\frac{\partial u_{p}^{i}}{\partial m^{2}} \cdot \frac{\partial u_{a}^{i} / \partial s^{j}}{\partial u_{a}^{2} / \partial m^{i}}}
$$

whose r.h.s. equals the r.h.s. of ( 3 ). Hence, in a SPE this sort of indifference curve for $p^{i}$ is tangent to the rival agent $a^{j}$ 's best reply function, as it usually occurs in a Stackelberg equilibrium when $p^{i}$ is the Stackelberg leader. In delegation games this condition holds simultaneously for all principals, i.e. all principals act as Stackelberg leaders with respect to all agents.

The tangency condition derived is very useful to construct equilibria in specific examples. Consider the literature on managerial contracts in oligopoly: Fershtman and Judd (1987) and Sklivas (1987) consider a homogeneous product linear duopoly with demand curve $p=a-b Q$ and costs $C^{i}=c q^{i}$ and managers interested only in their salary. The contracts considered are linear in revenues $R^{i}$ and costs $C^{i}$.

$$
\mu^{i}(q)=\alpha^{i}+R^{i}(q)-\beta^{i} C^{i}\left(q^{i}\right)=\alpha^{i}+\left(a-\beta^{i} c\right) q^{i}-b q^{i} q^{j}-b\left(q^{i}\right)^{2}
$$


The slope of agent's best reply function is $-1 / 2$ and the principal is able only to shift its intercept through the parameter $\beta^{i}$. Since the slope of the isoprofit curve is $\left(a-c-2 b q^{i}-b q^{j}\right) / b q^{i}$, the tangency condition is met only at one point, which is $q^{i}=q^{j}=2(a-c) / 5 b$.

Polo and Tedeschi (1992) consider in the same setting a class of contracts with relative performance

$$
\begin{aligned}
\mu^{i}(q) & =\alpha^{i}+R^{i}(q)-\beta^{i} C^{i}\left(q^{i}\right)+\gamma^{i} \Pi^{j}(q) \\
& =\alpha^{i}+\left(a-\beta^{i} c\right) q^{i}-\left(b+\gamma^{i}\right) q^{i} q^{j}-b\left(q^{i}\right)^{2}-\gamma^{i} b\left(q^{j}\right)^{2}+(a-c) \gamma^{i} q^{j}
\end{aligned}
$$

It is easy to check that now the slope of the agent's best reply function is $-\left(b+\gamma^{i}\right) / 2 b$ : the principal is now able to choose the intercept and the slope of her agent's reaction function, and the tangency condition can be met at any individually rational allocation.

The above analysis can be used to prove a Folk Theorem for SPE in delegation games.

Theorem 2 (Folk Theorem): All the individually rational allocations $S_{0}$ are implementable as subgame perfect equilibrium outcomes with differentiable contracts.

Proof: Fix an allocation $\hat{s} \in S_{0}$ and the corresponding money transfers $\hat{m}$ such that $u_{a}^{i}\left(\hat{s}, \hat{m}^{i}\right)=\bar{u}_{a}$. The values of $u_{p}^{i}$ and $u_{a}^{i}$ and their respective derivatives of all orders are determined at $(\hat{s}, \hat{m})$. Take any pair of differentiable compensation functions $\breve{\mu}^{i}(s)$, suppose that $\hat{s}$ is an internal maximum ${ }^{7}$ and evaluate the restrictions on the compensation functions that must be met in agents' and principals' problems. From agents' problem we obtain three conditions:

$$
\begin{aligned}
\breve{\mu}(\hat{s}) & =\hat{m} \\
\frac{\partial u_{a}^{i}}{\partial s^{i}}+\frac{\partial u_{a}^{i}}{\partial m^{i}} \cdot \frac{\partial \breve{\mu}^{i}}{\partial s^{i}} & =0 \\
\frac{\partial^{2} u_{a}^{i}}{\partial s^{i 2}}+\frac{\partial^{2} u_{a}^{i}}{\partial m^{i} \partial s^{i}} \cdot \frac{\partial \breve{\mu}^{i}}{\partial s^{i}}+\frac{\partial u_{a}^{i}}{\partial m^{i}} \cdot \frac{\partial^{2} \breve{\mu}^{i}}{\partial s^{i 2}} & <0
\end{aligned}
$$

For agent $i,(5)$ determines the intercepts of the contract, (6) determines the value of $\partial \breve{\mu}^{i} / \partial s^{i}$, while ( 7 ) sets restrictions on the possible values of $\partial^{2} \breve{\mu}^{i} / \partial s^{i 2}$. The principals' programs impose further restrictions on the admissible compensation schemes. The first order conditions are summarized for each principal in equation ( 3 ). Since the r.h.s. of $(3)$ is a number at the proposed allocation, from principal $j$ 's program we obtain a restriction on the slope of agent $i$ 's best reply $\partial \tilde{\sigma}^{i} / \partial s^{j}$. Totally differentiating ( 6 ) we obtain:

\footnotetext{
${ }^{7}$ This is not restrictive since, given our assumptions on the utility function of agents and principals, the tangency condition we are using can be implemented even at the boundary of $S_{0}$ for right or left derivatives. Hence by focussing on internal maxima we are able to map all the relevant allocations.
} 


$$
\frac{\partial \tilde{\sigma}^{i}}{\partial s^{j}}=-\frac{\frac{\partial^{2} u_{a}^{i}}{\partial s^{2} \partial s^{j}}+\frac{\partial^{2} u_{a}^{i}}{\partial m^{2} \partial s^{j}} \cdot \frac{\partial \breve{\mu}^{i}}{\partial s^{2}}+\frac{\partial u_{a}^{i}}{\partial m^{i}} \cdot \frac{\partial^{2} \breve{\mu}^{i}}{\partial s^{2} \partial s^{j}}}{\frac{\partial^{2} u_{a}^{i}}{\partial s^{2}}+\frac{\partial^{2} u_{a}^{i}}{\partial m^{2} \partial s^{i}} \cdot \frac{\partial \breve{\mu}^{i}}{\partial s^{i}}+\frac{\partial u_{a}^{i}}{\partial m^{2}} \cdot \frac{\partial^{2} \breve{\mu}^{i}}{\partial s^{22}}}
$$

which has to be equated to the r.h.s. of ( 3 ). Hence the principals' first order conditions can be met by choosing suitable values of $\partial^{2} \breve{\mu}^{i} / \partial s^{i 2}$ and $\partial^{2} \breve{\mu}^{i} / \partial s^{i} \partial s^{j}$. Finally, the second order conditions for principals' program set restrictions on the sign of the determinant of the Hessian matrix, which is a function of $\partial^{2} \tilde{\sigma}^{i} / \partial s^{j 2}$, which in turn can be set at the desired level through the terms $\partial^{3} \breve{\mu}^{i} / \partial s^{i 2} \partial s^{j}, \partial^{3} \breve{\mu}^{i} / \partial s^{i} \partial s^{j 2}$ and $\partial^{3} \breve{\mu}^{i} / \partial s^{i 3}$. A pair of contracts with polynomial form can always meet all the restrictions on the partial derivatives and on the intercept induced by the first and second order conditions of the agents' and principals' problems, which were just described. So we know that the initial allocation $(\hat{s}, \hat{m})$ is a local maximum for the agents, while $\breve{\mu}(s)$ is a local maximum for the principals. We can always find a polynomial function which satisfies the second order conditions globally in $S$.

Theorem 2 states that, in general, any equilibrium ourcome $\hat{s}$ consistent with the individual rationality constraints can be implemented as a SPE in a delegation game. This result resembles, in a static setting, the well known Folk Theorem in repeated games. The multiplicity of equilibria is endemic in delegation games and derives from two different features of the model; first of all, the equilibrium conditions set only local restrictions on the compensation schemes of the agents at a specific equilibrium point, and are consistent with many different contracts associated to the same equilibrium outcome $\hat{s}$. Secondly, the principals are able to implement many different equilibrium outcomes by selecting the appropriate compensation schemes. We have therefore a multiplicity of equilibria in both the contract and the action spaces ${ }^{8}$.

\section{Renegotiation}

It has been argued - Katz (1991) - that admitting the possibility of renegotiating the contracts has the same effects on delegation games as the assumption of non observ-

\footnotetext{
${ }^{8}$ Our result, as well as the nature of the multiplicity of equilibria and the crucial conditions for an equilibrium, share some features of the literature on competition in supply functions - Klemperer and Meyer (1989). In that setting, firms compete in the market by committing to a supply schedule, while in equilibrium each firm maximizes profits given its residual demand, which is determined by the rival's supply function. The main difference between the supply function approach and our own is that in our model each principal must take into account her agent's preferences and participation constraint in order to implement an action through an incentive scheme. The participation constraint implies that the set of equilibrium allocations might be smaller in a delegation game than in a supply function model, while agent's preferences impose stronger restrictions on the set of contracts which can implement a particular allocation in delegation as compared to supply function models. In many applications, e.g. managerial contracts and retailer-producers relations, agents' utility does not depend directly upon actions and their outside option is the same as that of the principals. In these cases we expect a one-to-one relation between delegation and supply function equilibria. For similar results in an asymmetric information model see Kühn (1990).
} 
able contracts, that is it reduces the strategic effect of delegation. With asymmetric information Katz (1991) and Caillaud et al. (1993) prove that delegation can still have strategic effects under specific assumptions, even if secret renegotiation is admitted, but the Folk Theorem result does not hold anymore. In this section we shall show that the consequences of renegotiation are not necessarily the same as those of non observable contracts, even with symmetric information. More precisely, renegotiation reduces the possibilities for the strategic use of delegation only when the side and the main contracts are perfect substitutes for the principals. If, on the contrary, there is a (slightly) higher marginal cost in compensating the agent through a side contract, then delegation induces the large multiplicity of equilibria described in section $3^{9}$. If, instead, renegotiation implies a lump sum dead-weight loss, delegation still enlarges the set of equilibrium allocations, but in general a Folk Theorem does not hold.

The formal assumptions of the model are as follows. All the assumptions from 1 to 5 are almost the same as in section 2. That amounts to saying that we have two agencies each composed by one principal and one agent. The agents' sets of actions are interval in $\Re$, as in the previous sections. The agents' utility functions have the same properties and players face the same individual rationality constraints as in section 2 . The only modification refers to the utility function of the principals.

Assumption $3 \mathrm{r}$ (Principals' utility). $u_{p}^{i}: S \times \Re \times \Re \rightarrow \Re^{+}$is the utility function of $p^{i}$ with the following properties: $u_{p}^{i} \in C^{2}$, it is strictly quasi-concave in $\left\{s^{i}, m_{m}^{i}, m_{s}^{i}\right\}$ and strictly decreasing in the compensations determined by the main contract, $m_{m}^{i}$, and by the side contract, $m_{s}^{i}$. Total compensation is the sum of the main and of the side compensations, i.e. $m^{i}=m_{m}^{i}+m_{s}^{i}$. Moreover we assume that $\partial u_{p}^{i} / \partial m_{s}^{i}<\partial u_{p}^{i} / \partial m_{m}^{i}$ for $\forall s \in S$ and any value of $m^{i}$.

The main difference with respect to Assumption 3 is that $3 \mathrm{r}$ specifies different marginal costs to the principal for the main and the side contracts. In particular there is a cost in deviating from the main contract represented by the assumption about the derivatives of $u_{p}^{i}$ with repect to the main and the side compensations ${ }^{10}$. Transferring one unit of money to the agent through the side contract costs more to the principal in utility terms than through the main contract. There are various justifications of this assumption, broadly related to the cost of keeping secret the contract. Higher costs can depend upon internal organization reasons, upon capital market imperfections that make financing the side transfer more costly, or can derive from the use of non monetary transfers. In a word, these renegotiation costs are additional transaction costs ${ }^{11}$.

\footnotetext{
${ }^{9}$ Hence, we focus on side transfer technologies that imply a dead-weight loss increasing in the size of the secret transfer of money from the principal to the agent. Transfer costs play a crucial role also in the theory of interest groups and regulation (Stigler (1971)) and in the analysis of collusion in agency relations (Tirole (1992)). In these cases the dead-weight loss derives from the use of some "income equivalent" instead of money in the side transfer and again it is increasing in the size of the transfer.

${ }^{10}$ It could be objected that we do not consider principal's deviations with a negative side transfer. It will be argued that this is not a relevant deviation when checking for renegotiation proofness.

${ }^{11}$ An alternative assumption could be that the side transfer implies a lump sum dead-weight loss. The
} 
Finally, the utility function of the agent has the form $u_{a}^{i}\left(s, m_{m}^{i}+m_{s}^{i}\right)$. Therefore, side and main contracts are perfect substitutes for the agent. Assumption $6 \mathrm{r}$ adapts the timing of the game to the present three stage case.

Assumption $6 \mathbf{r}$ (Timing of the game). The utility functions of all principals and all agents are common knowledge from the beginning.

$t_{1}$ : Each principal proposes a main contract $\mu_{m}^{i}$ to the agent who can accept it or not; the main contract is a function $\mu_{m}^{i}: S \rightarrow \Re$. If $a^{i}$ refuses it, the game is over, otherwise if continues;

$t_{1}^{\prime}:$ All contracts become public information;

$t_{2}$ : Each $p^{i}$ proposes a side contract $\mu_{s}^{i}$ to the agent who can accept it or not; the side contract is a function $\mu_{s}^{i}: S \times M_{m} \rightarrow \Re$, where $M_{m}$ is the set of principals' main contracts offered ad $t_{1}$. If $a^{i}$ refuses, then only the main contract is enforceable, otherwise they are both enforceable, i.e. the total compensation is $m^{i}=m_{m}^{i}+m_{s}^{i}$, where $m_{m}^{i}=\mu_{m}^{i}(s)$ and $m_{s}^{i}=\mu_{s}^{i}(s)$.

$t_{3}:$ Each $a^{i}$ chooses an action $s^{i} \in S^{i}$

$t_{4}: s$ is observed (and verifiable) and all contracts are implemented.

The crucial difference between the main and the side contract is that while the former becomes public information, the latter is privately observed by $a^{i}$ and $p^{i}$. The effects of assuming $\partial u_{p}^{i} / \partial m_{s}^{i}<\partial u_{p}^{i} / \partial m_{m}^{i}$ might be now better understood. If the two derivatives were equal, there would not be any difference between the main and the side contracts, that is, the principals would be able to counterbalance the main contract with the side payment, obtaining in $t_{2}$ any desired net payment to the agent with no additional cost. This is no longer the case if the side transfer is more costly to the principal than the main contract.

In the present set up we have also to modify the assumptions referring to players' strategies.

Assumption $7 \mathbf{r}$ (Principals' strategy). $p^{i}$ in $t_{1}$ chooses a main contract $\mu_{m}^{i}: S \rightarrow \Re$. The set of all such functions is $M_{m}^{i}$ and that of principals' strategy profile in $t_{1}$ is $M_{m}=M_{m}^{1} \times M_{m}^{2} \cdot p^{i}$ in $t_{2}$ chooses a side contract $\mu_{s}^{i}: S \times M_{m} \rightarrow \Re$. The set of all such functions is $M_{s}^{i}$ while $M_{s}$ is the corresponding set of strategy profile.

reason why we are assuming transaction costs increasing in the deviation is twofold. On the one hand, the case of increasing transaction costs allows us to reach more precise and better characterized results with respect to the lump sum case. Hence, we deal with the former more extensively. On the other hand, on a theoretical ground, it is not clear to us why the lump sum case should be more relevant than the alternative one, as a representation of the costs of keeping secret the side contract. While a precise assessment of which is the correct assumption can be done only empirically and case by case, it is here convenient to analyze more in details the assumption which allows us to derive stringer results. 
Assumption 8r (Agents' strategy). A strategy for agent $a^{i}$ is the choice of a function $\sigma^{i}: M_{m} \times M_{s} \rightarrow S^{i}$. $\Sigma^{i}$ is the strategy set of agent $a^{i}$ and the set of all agents' strategy profiles is $\Sigma=\Sigma^{1} \times \Sigma^{2}$, with generic element $\sigma$.

Given Assumptions 1, 2, 3r, 4, 5, 6r, 7r, and 8r, a three stage delegation game with renegotiation is

$$
{ }_{r}^{d}=\left\{\{P, A\},\left\{M_{m}, M_{s}, \Sigma\right\}\left\{u_{p}^{i}, u_{a}^{i}\right\}_{i=1,2},\left\{\bar{u}_{p}, \bar{u}_{a}\right\}\right\}
$$

\section{Existence of a Renegotiation Proof Equilibrium}

Subgame perfection remains the appropriate equilibrium concept also in this modified setting, given the time and information structure described in Assumption 6r. The definition of subgame perfection in this new set up is easily derived from that of section 3 . Before stating the main results, it is necessary fo define a Renegotiation proof equilibrium (RPE). An equilibtium $\left\{\hat{\mu}_{m}, \hat{\mu}_{s}, \hat{\sigma}\right\}$ is said to be renegotiation proof if it is subgame perfect and if $\hat{\mu}_{s}\left(\hat{\mu}_{m}(s), s\right)=0, \forall s \in S$. The definition of renegotiation proofness in the present context is quite obvious: the contract must satisfy the proposed equilibrium concept and no principal proposes and/or no agent accepts a side contract.

Theorem 3 The set $\hat{S}_{r}$ of outcomes induced as an RPE of ${ }_{r}^{d}$ when $\hat{\mu}_{m}$ is differentiable and such that it induces internal maxima for the agents' problem is generically of zero measure.

Proof: Consider the last stage game, where the main contract $\hat{\mu}_{m}^{i}(s)$ is already chosen and the side contract $\mu_{s}^{i}$ is to be determined for each agency. Let $\hat{s}$ be the allocation and $\hat{m}_{m}$ be the set of money transfers that emerge in the agents' game equilibrium if no side contract is offered, i.e. if $\mu_{s}^{i}(s)=0, \forall s \in S, i=1,2$. Principal $p^{i}$ s problem (R) when choosing the side contract is

$$
\begin{array}{ll} 
& \max _{m_{s}^{i}, s^{i}} u_{p}^{i}\left(s^{i}, \hat{s}^{j}, \hat{\mu}_{m}^{i}\left(s^{i}, \hat{s}^{j}\right), m_{s}^{i}\right) \\
\text { s.t. } & u_{a}^{i}\left(s^{i}, \hat{s}^{j}, \hat{\mu}_{m}^{i}\left(s^{i}, \hat{s}^{j}\right)+m_{s}^{i}\right) \geq \bar{u}_{a}
\end{array}
$$

The first order conditions, evaluated at $m_{s}^{i}=0$ and $s^{i}=\hat{s}^{i}$ are

$$
\begin{aligned}
\frac{\partial u_{p}^{i}}{\partial s^{i}}+\frac{\partial u_{p}^{i}}{\partial m_{m}^{i}} \cdot \frac{\partial \hat{\mu}_{m}^{i}}{\partial s^{i}}+\lambda^{i}\left(\frac{\partial u_{a}^{i}}{\partial s^{i}}+\frac{\partial u_{a}^{i}}{\partial m^{i}} \cdot \frac{\partial \hat{\mu}_{m}^{i}}{\partial s^{i}}\right) & =0 \\
\frac{\partial u_{p}^{i}}{\partial m_{s}^{i}}+\lambda^{i} \frac{\partial u_{a}^{i}}{\partial m^{i}} & =0
\end{aligned}
$$


where $\lambda^{i}$ is the Lagrange multiplier of the agent's participation constraint in problem $(\mathrm{R})$. Since by assumption we must be at an equilibrium in the agents' game with differentiable contracts, the following holds:

$$
\frac{\partial u_{a}^{i}}{\partial s^{i}}+\frac{\partial u_{a}^{i}}{\partial m^{i}} \cdot \frac{\partial \hat{\mu}_{m}^{i}}{\partial s^{i}}=0
$$

which, together with (9), implies

$$
\frac{\partial u_{p}^{i}}{\partial s^{i}}+\frac{\partial u_{p}^{i}}{\partial m_{m}^{i}} \cdot \frac{\partial \hat{\mu}_{m}^{i}}{\partial s^{i}}=0
$$

On the other hand, solving (11) and (12) for $\partial \mu_{m}^{i} / \partial s^{i}$ and equating them, we obtain

$$
\frac{\partial u_{p}^{i} / \partial s^{i}}{\partial u_{p}^{i} / \partial m_{m}^{i}}=\frac{\partial u_{a}^{i} / \partial s^{i}}{\partial u_{a}^{i} / \partial m^{i}}
$$

For generic utility functions $u_{p}^{i}$ and $u_{a}^{i},(13)$ and the participation constraint of the agent are satisfied simultaneously with equality sign only in isolated points. At these allocations $p^{i}$ has no incentive to offer a side contract, i.e. the main contract and the agents' choices are RPE. Finally, a suitable choice of $\lambda^{i}$ solves (10), which therefore does not pose any further restriction.

Notice that in problem $(\mathrm{R})$ we use the rival agent's action $\hat{s}^{j}$, and not his best reply function $\tilde{\sigma}^{j}$, because a deviation of $p^{i}$ from a RPE is not observed by the other agency and therefore the new best reply function chosen by agent $a^{i}$ cannot be conjectured by the other agent. For the same reason, the term $\frac{\partial u_{p}^{i}}{\partial s^{3}} \frac{\partial \tilde{\sigma}^{j}}{\partial s^{2}}$ does not appear in (9). This is formally the main implication of the assumption of non observable side contracts. Furthermore, in the models in which agents' utility does not depend upon the actions but only on the payment, as usually assumed in the managerial contracts literature, at an agents' equilibrium $\partial u_{a}^{i} / \partial s^{i}=0$ and (13) implies $\partial u_{p}^{i} / \partial s^{i}=0$, which correspond to the first order conditions of a game in which the principals play directly the game. In this case, with differentiable main contracts, the game with unobservable side contracts and that without delegation have the same subgame perfect equilibrium outcomes.

Theorem 3 implies that a necessary condition for an outcome not belonging to $\hat{S}_{r}$ to be sustained as RPE is that the main contract of each agency $\mu_{m}^{i}(s)$ has a kink at the equilibrium outcome. The next theorem shows that the entire set of individually rational outcomes can be sustained as a RPE by such contracts.

Theorem 4 All the individually rational allocations $S_{0}$ can be implemented as $R P E$ outcomes in ${ }_{r}^{d}$. If $\hat{s} \notin \hat{S}_{r}$ (the set of RPE defined in Theorem 3) the main contracts of all agencies, $\hat{\mu}_{m}(s)$, must be non differentiable at $\hat{s}$.

Proof: The proof will be constructive. Suppose that the strategy profile $(\hat{\sigma}, \hat{\mu})$ with outcome $(\hat{s}, \hat{m})$ is a SPE with differentiable contracts in the delegation game without 
renegotiation, ${ }^{d}$ with the same players and utility functions as in,${ }_{r}^{d}$. We know from Theorem 2 that $(\hat{\sigma}, \hat{\mu})$ exists for any $\hat{s} \in \hat{S}$. Now consider,${ }_{r}^{d}$ and set $\mu_{m}^{i}=\hat{\mu}^{i}$. Let's consider two cases.

a) At the allocation $\hat{s}$

$$
\frac{\partial u_{p}^{i}}{\partial s^{i}}+\frac{\partial u_{p}^{i}}{\partial m^{i}} \cdot \frac{\partial \mu_{m}^{i}}{\partial s^{i}}>0
$$

(14) and (11) imply that (9) is positive, i.e. the principal has an incentive to induce an increase in $s^{i}$ through secret renegotiation. Therefore for $s^{i} \leq \hat{s}^{i}$ the proposed main contract does not pose any problem. Let's consider whether we can modify the contract in order to make it renegotiation proof also for $s^{i}>\hat{s}^{i}$. If we choose a main contract $\tilde{\mu}_{m}^{i}$ which satisfies (9) as an equality for $m_{s}^{i}=0$ and $s^{i}=\hat{s}^{i}$, the principal has no incentive to offer a side contract, provided that the second order conditions are globally satisfied. Moreover, from (9) and (10) we obtain

$$
\left(1-\frac{\partial u_{p}^{i} / \partial m_{m}^{i}}{\partial u_{p}^{i} / \partial m_{s}^{i}}\right) \cdot \frac{\partial \tilde{\mu}_{m}^{i}}{\partial s^{i}}=\frac{\partial u_{p}^{i} / \partial s^{i}}{\partial u_{p}^{i} / \partial m_{s}^{i}}-\frac{\partial u_{a}^{i} / \partial s^{i}}{\partial u_{a}^{i} / \partial m^{i}}
$$

Still, from (9) and (10) it derives

$$
\left(1-\frac{\partial u_{p}^{i} / \partial m_{m}^{i}}{\partial u_{p}^{i} / \partial m_{s}^{i}}\right) \cdot \frac{\partial \hat{\mu}^{i}}{\partial s^{i}}>\frac{\partial u_{p}^{i} / \partial s^{i}}{\partial u_{p}^{i} / \partial m_{s}^{i}}-\frac{\partial u_{a}^{i} / \partial s^{i}}{\partial u_{a}^{i} / \partial m^{i}}
$$

which implies that $\partial \hat{\mu}^{i} / \partial s^{i}>\partial \tilde{\mu}_{m}^{i} / \partial s^{i}$. This, in turn, implies that

$$
\frac{\partial u_{a}^{i}}{\partial s^{i}}+\frac{\partial u_{a}^{i}}{\partial m^{i}} \cdot \frac{\partial \tilde{\mu}_{m}^{i}}{\partial s^{i}}<0
$$

in a right neighborhood of $\hat{s}^{i}$ and consequently $\hat{s}^{i}$ is still the optimal action for the agent. It is obviously always possible to choose a $\tilde{\mu}_{m}^{i}$ is such a way that the second order conditions are globally satisfied. Consequently, the main contract

$$
\hat{\mu}_{m}^{i}(s)= \begin{cases}\tilde{\mu}_{m}^{i}(s) & \text { for } s^{i}>\hat{s}^{i} \text { and } s^{j}=\hat{s}^{j} \\ \hat{\mu}^{i}(s) & \text { otherwise }\end{cases}
$$

is renegotiation proof and induces $\hat{s}$ as a SPE outcome.

b) In the non renegotiation proof SPE, at the allocation $\hat{s}$

$$
\frac{\partial u_{p}^{i}}{\partial s^{i}}+\frac{\partial u_{p}^{i}}{\partial m_{m}^{i}} \cdot \frac{\partial \hat{\mu}_{m}^{i}}{\partial s^{i}}<0
$$

Then for $s^{i} \geq \hat{s}^{i}$ the non renegotiation proof contract can still be used. For $s^{i}<\hat{s}^{i}$ the proof can be easily adapted from that of case a) above.

The proof of the Theorem offers a clear insight on how a RPE works. We have seen that in general the principal has a first order incentive to secretly induce a deviation, if 
she uses a differentiable main contract. In fact, in a secret renegotiation the principal has to maintain the agent at his reservation utility $\bar{u}_{a}$, by compensating the agent through the side transfer $m_{s}^{i}$. But with a smooth main contract, at $\hat{s}$, the agent has only second order effects in deviating from $\hat{s}^{i}$, and therefore he requires only second order modifications of the side transfer in order to remain indifferent. Hence, if the main contract is smooth at $\hat{s}$, the principal can profitably deviate. By designing the main contract kinked at the proposed allocation, we can induce first order effects of the desired magnitude if the agent deviates. The relative inefficiency of using the side contract enables to make it unprofitable to offer the side contract to induce a deviation ${ }^{12}$. In summary a renegotiation proof main contract must counterbalance the principal's incentive to deviate by making very costly to the agent to comply to the principal's desire. According to such a contract, the agent requires a high compensation in order to deviate; since this latter must be payed inefficiently through the side transfer, inducing the deviation becomes unprofitable for the principal. The two ingredients that allow to obtain this result are a kink in the main contract $\tilde{\mu}_{m}^{i}$ at the desired action $\hat{s}^{i}$ and a higher marginal cost of the side contract for the principal.

If renegotiation implies a lump sum dead-weight loss, when offering a side contract the principal compares her first order increase in utility to the lump sum cost and the extra payment due to her agent. This payment has only second order effects on principal's utility, since there is always a smooth contract which implements the desired action. It is intuitive that renegotiation will occur if the lump sum dead-weight loss is lower than the increase in utility the principal can obtain by inducing the agent's deviation. Large lump sum losses will prevent most of the possible deviations, thus making a large set of allocations to be renegotiation proof equilibrium outcomes. However, small lump sum costs would be insufficient to prevent renegotiation in a large set of allocations. In all cases, it will be true that the set of implementable allocations has a positive measure, event though the Folk Theorem cannot hold anymore, unless in the special case of very large transaction costs. A precise characterization of the set of renegotiation proof equilibria cannot be obtained in this case without further restrictions on players' preferences.

\section{Concluding remarks}

Delegation games are defined by a formal structure which enables to analyze a variety of economic and policy design problems, such as managerial contracts in oligopoly, vertical relations among retailers and producers, strategic trade policies, the selection of political representatives in international institutions, ets. We observe an increasing use of this framework in the literature, that has been developed mainly through examples and

\footnotetext{
${ }^{12}$ Note that a deviation with a negative side transfer is not relevant when checking for renegotiation proofness. In fact, in a RPE the agent is at his preferred action according to the main contract, Therefore, if the principal whishes to induce a different action, she has to compensate her agent for the welfare loss with a positive money transfer.
} 
applications. Only a limited number of papers, instead, deal with general definitions and results.

In this paper we proved the existence and characterized the set of equilibria in delegation games in a unified formal structure, which can encompass most of the applications in the literature. In this framework we prove a Folk Theorem which is more general than that obtained by Fershtman, Judd and Kalai (1991) since we allow for non separable utilities. Moreover, the set of equilibrium outcomes includes all the individually rational allocations and not only the Pareto efficien ones, as in the quoted paper. In proving the Folk Theorem we derive a useful feature of equilibria in delegation games, which we define the Stackelberg Property. This property states that in a subgame perfect equilibrium each principal chooses a contract that induces the most preferred allocation on the reaction function of the rival agent, once taken into accounto the participation contraint of her own agent. Therefore, each principal acts as a Stackelberg leader with respect to all agents. In the text we argued why this property can explain the high multiplicity of equilibria, which is endemic in delegation games. Moreover, the Stackelberg Property allows to find a simple algorithm to construct equilibria in specific examples.

The second part of the paper deals with the renegotiation problem. It is often argued that contract renegotiation, as contract non observability, reduces the possibilities of a strategic use of delegation. The existing literature found that strategic effects of delegation persist only with asymmetric information, even when renegotiation is allowed. However, a Folk Theorem was not proved as yet. In a symmetric information setting we studied secret renegotiation assuming that the side contract implies a dead-weight loss. When this additional cost of renegotiation is increasing in the size of the side payment, we proved that a Folk Theorem still holds, provided that the main contracts are kinked at the desired allocation. If the dead-weight loss is lump sum, a strategic effect of delegation still exists, but the set of renegotiation proof allocations is smaller.

\section{References}

[1] Brander J, Spencer P. (1983), International RD Rivalry and Industrial Strategy, Review of Economic Studies, 50, 707-22.

[2] Brander J, Spencer P. (1985), Export Subsidies and International Market Share Rivalry, Journal of International Economics, 18, 83-100.

[3] Caillaud B., Jullien B., Picard P. (1993), Competing Vertical Structures and Renegotiation, Econometrica, 63, 621-46.

[4] Eaton J. Grossman G. (1986), Optimal Trade and Industrial Policy Under Oligopoly, Quarterly Journal of Economics, 101, 383-406.

[5] Fershtman C., Judd K., (1986), Strategic Incentive Manipulation in Rivalrous Agencies, WP IMSSS Stanford. 
[6] Fershtman C., Judd K., (1987), Equilibrium Incentives in Oligopoly, American Economic Review, 927-40.

[7] Fershtman C., Judd K., Kalai E. (1991), Observable Contracts: Strategic Delegation and Cooperation, International Economic Review, 3, 551-60.

[8] Katz M. (1991), Game Playing Agents: Unobservable Contracts as Precommitments, Rand Journal of Economics, 22, 307-28.

[9] Klemperer P. Meyer M. (1989), Supply Function Equilibria in Oligopoly under Uncertainty, Econometrica, 57, 1243-77.

[10] Koray S. Sertel M. (1989), Limit Theorems for Recursive Delegation Equilibria, mimeo.

[11] Kühn K. (1990), Vertical Restraints in a Manifacturer Duopoly: the Case of Non Linear Pricing, mimeo.

[12] Laussel D. (1992), Strategic Commercial Policy Revisited: a Supply Function Equilibrium Model, American Economic Review, 82, 84-99.

[13] Maggi G. (1991), Strategic Trade Policy under Asymmetric Information, mimeo.

[14] Persson T. Tabellini G. (1992), The Politics of 1992: Fiscal Policy and European Integration, Review of Economic Studies, 59, 689-701.

[15] Polo M. Tedeschi P. (1990), Managerial Contracts and the Divisional Structure of the Firm, mimeo.

[16] Polo M. Tedeschi P. (1992), Managerial Contracts, Collusion and Mergers, Ricerche Economiche, 46, 281-302.

[17] Sklivas S. (1987), The Strategic Choice of Managerial Incentives, Rand Journal of Economics, 4, 473-86.

[18] Stigler G. (1971), The Economic Theory of Regulation, Bell Journal of Economics, $2,3-21$.

[19] Tirole J. (1992), Collusion and the Theory of Organization, in Laffont J.J. (ed.) Advances in Economic Theory, Sixth World Congress, Vol. II, Cambridge, Cambridge U.P.

[20] Vickers J. (1985), Delegation and the Theory of the Firm, Economic Journal Supplement, $95,138-47$. 